$54^{\text {ème }}$ Congrès de la SFMBCB, 03016 (2011)

DOI: $10.1051 / \mathrm{sfmbcb} / 20115403016$

(C) Owned by the authors, published by EDP Sciences, 2011

\title{
Ingénierie tissulaire osseuse et cellules souches mésenchymateuses : applications à la sphère buccale et maxillo-faciale
}

\author{
Bouvet-Gerbettaz $\mathbf{S}^{1,2}$, Carle GF ${ }^{1}$, Rochet $\mathbf{N}^{1}$ \\ ${ }^{1}$ Laboratoire GéPITOs, Université de Nice Sophia-Antipolis, CNRS, UFR Médecine, Nice, France \\ ${ }^{2}$ UFR d'Odontologie, Service d'Odontologie, $\mathrm{CHU}$, Nice, France \\ sbouvet@unice.fr
}

Les cellules souches mésenchymateuses adultes ont suscité un engouement important dans le milieu de la recherche biomédicale (Bianco et al. 2008). A ne pas confondre avec les cellules souches embryonnaires dont l'usage est prohibé en France pour des raisons éthiques, les cellules souches mésenchymateuses adultes ont fait l'objet de nombreuses études in vitro et in vivo qui ont permis de caractériser leurs propriétés uniques de prolifération et de différentiation, et donc potentiellement de régénération tissulaire. Grâce à une exploration systématique des différents tissus mésenchymateux adultes, il a été possible d'isoler ces cellules souches mésenchymateuses à partir de la mœlle osseuse, de tissu adipeux, de sang périphérique ou même de la pulpe dentaire (Cancedda et al. 2007). Ces cellules ont un très fort taux de prolifération et sont capables de se différencier en plusieurs types cellulaires tels que les myoblastes, chondroblastes, ostéoblastes ou encore adipocytes.

Ces propriétés en font des candidats très intéressants pour la mise en place d'une thérapie cellulaire de certaines pathologies dégénératives, comme l'ostéonécrose des maxillaires, ou pour l'ingénierie tissulaire dans le domaine des pertes de substances osseuses acquises ou congénitales. Au travers d'une revue de la littérature (Schimming et al. 2004, Pradel et al 2006, Pradel et al. 2007), nous rapporterons des études précliniques et cliniques utilisant les cellules souches mésenchymateuses adultes dans la sphère maxillo-faciale. Nous comparerons ces résultats aux données obtenues avec des modèles d'ingénierie tissulaire développés au laboratoire (Elabd et al. 2007, Trojani et al. 2008).

Malgré de nombreux progrès, cette approche thérapeutique est encore souvent jugée trop lourde et trop coûteuse en raison des nombreux intervenants, du plateau technique ou des substances pharmacologiques nécessaires, ce qui limite malheureusement les retombées cliniques. Par ailleurs l'effet biologique de ces cellules souches mésenchymateuses après implantation, semblerait lié à l'expression in situ de nombreux facteurs paracrines. Plusieurs équipes essaient de les identifier, afin de pouvoir stimuler directement la réparation tissulaire et de simplifier les applications cliniques chez l'homme.

This is an Open Access article distributed under the terms of the Creative Commons Attribution-Noncommercial License 3.0, which permits unrestricted use, distribution, and reproduction in any noncommercial medium, provided the original work is properly cited. 